\title{
Controversies in the role of postmastectomy radiotherapy in breast cancer patients with one to three positive axillary nodes and safety of integrating radiotherapy and breast reconstruction
}

\author{
Kenji Sekiguchi ${ }^{1,2}$
}

Received: 5 June 2017/ Accepted: 8 June 2017/Published online: 14 June 2017

(C) The Japanese Breast Cancer Society 2017

In this special feature, readers will find four articles related to postmastectomy radiotherapy (PMRT).

In two articles the issue of whether to recommend PMRT to all patients with 1-3 positive nodes was discussed. The use of PMRT has been widely accepted for patients with four or more positive lymph nodes, but controversy has remained regarding the role of PMRT for those with 1-3 positive nodes. In 2014, the Early Breast Cancer Trialists' Collaborative Group (EBCTCG) published an updated meta-analysis of the effects of PMRT [1] which generated renewed interest in the value of PMRT. One of the main findings is that PMRT is not only highly effective at preventing locoregional recurrence (LRR) but also reduces the risk of distant metastases and breast cancer mortality in patients with 1-3 positive nodes. Among 1314 patients with 1-3 positive nodes, PMRT resulted in a 10-year absolute decrease in any first recurrence of $11.5 \%$ and a 20 -year decrease in breast cancer mortality of $7.9 \%$ (both $P \leq 0.01$ ). However, the issue of PMRT for patients with 1-3 positive nodes remains far from being resolved even in light of this meta-analysis. Many clinicians seem to not believe that the favorable outcomes seen in the metaanalysis are generalizable to all breast cancer patients with 1-3 positive nodes.

Following the publication of the EBCTCG meta-analysis (June 2014), the consensus panel of the 14th St. Gallen Breast Cancer Conference (March 2015) considered that

Kenji Sekiguchi

kenjisek@luke.ac.jp

1 Sonoda-kai Radiation Oncology Clinic, 3-4-19 Hokima, Adachi-ku, Tokyo 121-0064, Japan

2 Department of Radiation Oncology, St. Luke's International Hospital, 9-1, Akashi-cho, Chuo-ku, Tokyo 104-8560, Japan
PMRT should be standard for patients with 1-3 involved nodes and adverse pathology. However, in the absence of adverse pathology, these patients could be treated without PMRT, although a slim majority would include such treatment for patients aged $<40$ years (51\%) [2]. In Japan, the questionnaire distributed at around the same time revealed that PMRT is never delivered to patients with 1-3 positive nodes at $41 \%$ of the institutes; PMRT is applied all of the time only in a minority of institutions (7.7\%) [3].

It is of course very important to scrutinize the evidence provided through recent studies, even if it is of high quality via a systematic review and meta-analysis. Thus, the debate in this issue by Ishikawa et al. and Tada et al. surrounding the pros and cons of PMRT in the setting of 1-3 positive nodes is both highly attractive and very timely [4, 5].

It should be considered that systemic and radiotherapy standards have improved considerably during the course of many of the studies included in the meta-analysis. Systemic therapy has proved to be highly effective at reducing the risk of both distant relapse and LRR. The trials included in the meta-analysis were predominantly conducted in the 1970s and 1980s and CMF and/or tamoxifen was usually used at that time. The LRR rate in unirradiated patients with 1-3 positive nodes was $16.5 \%$ at 5 years, and was substantially higher than those seen today. Current systemic treatments usually include more effective strategies such as anthracycline and taxane chemotherapy, trastuzumab, and an aromatase inhibitor. Moreover, technological improvements and advances such as three-dimensional conformal radiotherapy in the delivery of PMRT have also made this approach more safe and effective.

Following the spectrum theory, we can easily understand the complex interaction between respective contributions of systemic and locoregional treatments to the final outcomes, including survival and toxic effects [6]. Once 
systemic therapy has effectively reduced the risk of distant metastasis and LRR, optimized locoregional control by eliminating residual microscopic foci aims to reduce the risk of secondary metastases and to contribute to an improved survival. With this perspective, radiation therapy can also be seen to elicit a systemic effect [7]. Thus, the biggest benefit seen for PMRT might be in patients with intermediate risk, rather than high risk, disease.

After the 2014 meta-analysis, three trials for mainly intermediate risk disease (MA.20 [8], EORTC 22922/10925 [9], DBCG-IMN [10]) demonstrated that regional nodal irradiation is associated with increased disease-free survival that can be attributed to a decrease in the risk of LRR and distant relapses. In addition, the results suggest an improved overall and breast cancer specific survival, showing no increase in other causes of death. More effective systemic therapies such as anthracycline \pm taxane and tamoxifen \pm aromatase inhibitor were used in those trials.

In 2016, the American Society of Clinical Oncology (ASCO) updated the guideline for the use of PMRT [11]. The panel unanimously agreed that the available evidence shows that PMRT reduces the risks of LRR, any recurrence, and breast cancer mortality for patients with 1-3 positive nodes. However, it was also recommended that PMRT should not be indicated in some subsets of patients with low risk of LRR, where potential toxicities outweigh the absolute benefit. However, it is fair to recognize that we cannot yet unambiguously define specific patient subgroups to which PMRT should not be administered. The decision to recommend PMRT or not requires a great deal of clinical judgment that depends on several factors. PMRT has been historically recommended based mainly on tumor size and the number of positive nodes, not considering the effect of systemic therapy and tumor subtype on local recurrence. The response to neoadjuvant chemotherapy and subtype also gives us more information for the risk of local recurrence. In addition, recent studies suggest that genomic profiling could reliably predict LRR after mastectomy and this approach may ultimately open the door to tailoring locoregional management strategies.

Further analyses of the combined results of all trials including the ongoing MRC/EORTC SUPREMO trial [12], and molecular subtype and genomic profiles translational research, are required to reliably discriminate patients who really benefit from PMRT among patients with 1-3 positive nodes.

In the remaining two articles, controversies regarding the safety of integrating radiotherapy and breast reconstruction were discussed. Recently, the number of patients who need PMRT and desire reconstruction is growing, and patients face challenging decisions that will impact not only their long-term disease control but also their quality of life. Adverse impact of PMRT on the breast with reconstruction has been widely feared, since PMRT is associated with increasing complications. However, PMRT should be applied regardless of breast reconstruction as it reduces the risk of LRR and breast cancer mortality in selected patients.

What remains questionable and controversial is what is predictive of a complication or whether treatment technique such as breast constructive procedure or timing is a determining factor. Answering this question is difficult, as many of the studies looking at the safety of integration of breast reconstruction and PMRT are of poor quality, and few show statistically significant data.

Sekiguchi et al. reviewed the issues related to breast reconstruction and PMRT from a radiation oncology perspective [13]. Autologous tissue appears to withstand damage by radiation better than implant-based reconstructions. Further, Terao et al. reported a retrospective single institution data of a relatively good outcome after breast reconstruction with autologous tissue and PMRT [14]. Their study makes a valuable contribution to our growing understanding of the importance of grafting a flap with a good blood supply. It is unfortunate that the manuscript from the plastic surgeon who is experienced in implant use could not be prepared this time.

In summary, in spite of a growing number of patients with breast reconstruction who need PMRT, optimal approaches to integrate PMRT and breast reconstruction are not well established thus far. More data are needed to help clarify this complex question. Additional data from prospective multicenter cohort studies including the MROC study [15] are therefore eagerly awaited.

Finally, when the role of PMRT is questioned, patients should be informed by high-quality evidence and undergo multidisciplinary preoperative evaluation by a radiation and medical oncologist and by a breast and plastic surgeon, as necessary. It remains extremely important to fully discuss with patients the potential benefits and possible toxicity from PMRT.

\section{References}

1. EBCTCG (Early Breast Cancer Trialists' Collaborative Group), McGale P, Taylor C, Correa C, Cutter D, Duane F, et al. Effect of radiotherapy after mastectomy and axillary surgery on 10-year recurrence and 20-year breast cancer mortality: meta-analysis of individual patient data for 8135 women in 22 randomised trials. Lancet. 2014;383:2127-35.

2. Coates AS, Winer EP, Goldhirsch A, Gelber RD, Gnant M, Piccart-Gebhart $\mathrm{M}$, et al. Tailoring therapies-improving the management of early breast cancer: St Gallen International Expert Consensus on the Primary Therapy of Early Breast Cancer 2015. Ann Oncol. 2015;26:1533-46. 
3. Yamauchi C, Komoike Y, Tsugawa K, Yotsumoto D, Wada N, Sugae S, et al. Questionnaire survey of axillary surgery and radiation therapy for breast cancer in Japan. The 28th Annual Meeting of the Japanese Society for Radiation Oncology. 2015.

4. Ishikawa T, Kaise H, Yamada K, Hosonaga M, Chishima T, Narui $\mathrm{K}$, et al. Objection to postoperative radiation therapy in breast cancer with one to three lymph nodes involvements. Breast Cancer. 2017. doi:10.1007/s12282-016-0749-5.

5. Tada K, Nishioka K, Kikuchi Y, Niwa T, Seto Y. Postmastectomy radiation therapy in breast cancer with 1-3 involved lymph nodes: the Pros. Breast Cancer. 2017. doi:10.1007/s12282-0170787-7.

6. Poortmans P. Postmastectomy radiation in breast cancer with one to three involved lymph nodes: ending the debate. Lancet. 2014;383:2104-6.

7. Harris JR. Fifty years of progress in radiation therapy for breast cancer. Am Soc Clin Oncol Educ Book. 2014;21-5.

8. Whelan TJ, Olivotto IA, Parulekar WR, Ackerman I, Chua BH, Nabid A, et al. Regional nodal irradiation in early-stage breast cancer. N Engl J Med. 2015;373:307-16.

9. Poortmans PM, Collette S, Kirkove C, Van Limbergen E, Budach $\mathrm{V}$, Struikmans $\mathrm{H}$, et al. Internal mammary and medial supraclavicular irradiation in breast cancer. $N$ Engl $J$ Med. 2015;373:317-27.

10. Thorsen LB, Offersen BV, Dano H, Berg M, Jensen I, Pedersen AN, et al. DBCG-IMN: a population-based cohort study on the effect of internal mammary node irradiation in early node-positive breast cancer. J Clin Oncol. 2016;34:314-20.

11. Recht A, Comen EA, Fine RE, Fleming GF, Hardenbergh PH, Ho AY, et al. Postmastectomy radiotherapy: an American Society of Clinical Oncology, American Society for Radiation Oncology, and Society of Surgical Oncology Focused Guideline Update. J Clin Oncol. 2016;34:4431-42.

12. Kunkler IH, Canney P, van Tienhoven G, Russell NS. Elucidating the role of chest wall irradiation in 'intermediate-risk' breast cancer: the MRC/EORTC SUPREMO trial. Clin Oncol (R Coll Radiol). 2008;20:31-4.

13. Sekiguchi K, Kawamori J, Yamauchi H. Breast reconstruction and postmastectomy radiotherapy: complications by type and timing and other problems in radiation oncology. Breast Cancer. 2017. doi:10.1007/s12282-017-0754-3.

14. Terao Y, Taniguchi K, Fujii M, Moriyama S. Postmastectomy radiation therapy and breast reconstruction with autologous tissue. Breast Cancer. 2017;91. doi:10.1007/s12282-017-0760-5.

15. Jagsi R, Momoh AO, Qi J, Hamill JB, Bilig J, Kim HM, et al. Impact of radiotherapy on complications and patient-reported outcomes after breast reconstruction: Findings from the prospective multicenter MROC study. San Antonio Breast Cancer Symposium. 2016. 